\title{
Growth and pubertal development of adolescent boys on stimulant medication for attention deficit hyperactivity disorder
}

\section{Alison S Poulton MA, MB BChir, MD Clinical Senior Lecturer \\ Elaine Melzer RN, RM, OAM, \\ Research Associate Department of Paediatrics and Child Health \\ Paul R Tait MB BS, FRACP, \\ Paediatrician ${ }^{2}$ \\ Sarah P Garnett \\ BSc, MNutrDiet, PhD, BSc, MNutrDiet, PhD, Department of Paediatrics and Child Health \\ Chris T Cowell MB BS, FRACP. Professor of Paediatrics and Child Health \\ Louise A Baur MB BS, PhD, FRACP Professor of Paediatrics and Child Health \\ Simon Clarke MB BS, FRACP, FCP Medical Director \\ Department of Adolescent Medicine}

1 Sydney Medica School Nepean, University of Sydney, Sydney, NSW.

2 The Children's Hospita at Westmead,

University of Sydney,

Sydney, NSW.

alison.poulton@ sydney.edu.au

MJA 2013; 198: 29-32 doi: 10.5694/mjal2.10931 w eight loss and attenuated growth in height in children being treated with stimulant medication for attention deficit hyperactivity disorder (ADHD) is an area that has been plagued by controversy. ${ }^{1}$ The question of treatment affecting pubertal development is perhaps even more emotive as delay may be associated with significant psychopathology. ${ }^{2}$

The aims of this study were to investigate the influence of stimulant medication on growth rates and pubertal attainment of adolescent boys with ADHD and to compare these with a contemporaneous local cohort of community-sampled boys without ADHD. ${ }^{3-5}$ We have previously shown growth rates to be maximally attenuated during the first year, with a trend towards normalisation over 3 years of treatment. ${ }^{1}$ This suggests a progressive effect of stimulant medication on growth that plateaus at 3 years. Therefore, in order to standardise the effect of stimulant medication in this study, the subjects had a minimum of 3 years of continuous treatment.

\section{Methods}

To monitor growth and development in boys receiving stimulant treatment for ADHD, we used cross-

\section{Outline of study design}

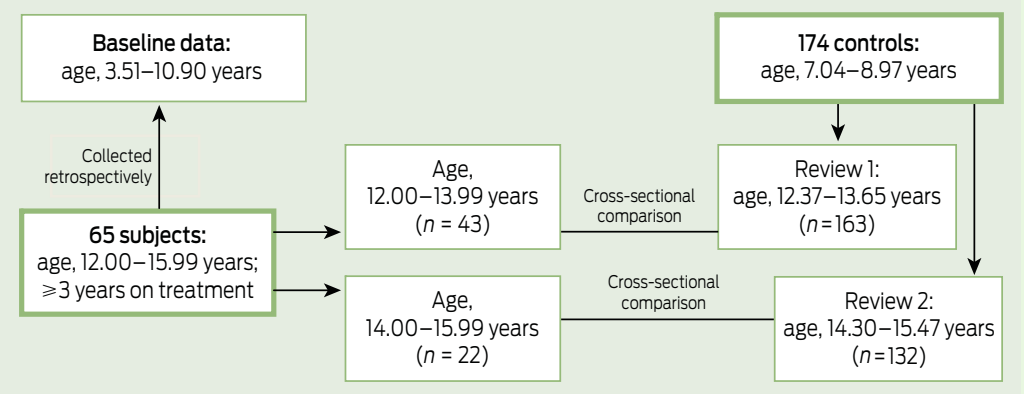

Objective: To investigate the growth and pubertal attainment of boys with attention deficit hyperactivity disorder (ADHD) on stimulant medication.

Design, setting and participants: Longitudinal study of boys aged 12.00-15.99 years at recruitment in 2005-2011, with stimulant-treated ADHD for at least 3 years, attending three paediatric practices (subjects), compared with longitudinal data from 174 boys from the Nepean longitudinal study (controls).

Main outcome measures: Subjects' growth parameters before treatment were compared with controls aged 7 or 8 years; growth parameters and longitudinal changes on treatment to ages 12.00-13.99 and 14.00-15.99 years were compared with controls reviewed at 13 and 15 years of age, respectively. The subjects' pubertal staging and height velocity were related to their treatment history.

Results: Sixty-five subjects were recruited; mean duration of treatment was 6.3 \pm 1.9 years. At baseline, their growth parameters were not significantly different from those of the controls after adjusting for age. Compared with the controls, after adjusting for current age and baseline growth parameter z score, subjects aged 12.00-13.99 years had significantly lower weight and body mass index $(P<0.01)$, and those aged 14.00-15.99 years had significantly lower height and weight $(P<0.05)$. At $12.00-13.99$ years of age, the subjects were comparable to the controls in their pubertal development adjusted for age, but those aged 14.00-15.99 years reported significant delay (mean Tanner stage, 3.6 for subjects $v 4.0$ for controls; $P<0.05$ ). The dose of medication was inversely correlated with the height velocity from baseline to 14.00-15.99 years of age $(P<0.05)$.

Conclusions: Prolonged treatment (more than 3 years) with stimulant medication was associated with a slower rate of physical development during puberty. To maintain adequate height velocity during puberty, we recommend keeping the dose as low as possible. sectional measures of anthropometry and pubertal stage in adolescence and of anthropometry in childhood, and compared these data with similar data from childhood and adolescence in boys without ADHD (Box 1). Having anthropometry at two time points for each child meant that growth velocities could also be compared.

For the ADHD group, adolescent boys aged 12.00-15.99 years with ADHD diagnosed according to Diagnostic and statistical manual of mental disorders, fourth edition, criteria and treated continuously with stimulant medication for at least 3 years, and who had pretreatment growth data available, were recruited from three paediatric practices in western Sydney between May 2005 and August 2011. AP recruited for the entire period; $\mathrm{SC}$ and $\mathrm{PT}$ recruited from February to May 2006 and S C from March to July 2007. Over this period, A P invited all eligible boys to participate; only two refused. SC and PT invited an estimated $84 \%$ and $50 \%$ of their eligible patients to participate 
2 Comparison of the growth and puberty data of subjects and controls, adjusting for age, using general linear modelling

\begin{tabular}{|c|c|c|c|}
\hline Variable & Subjects & Controls & \\
\hline \multicolumn{4}{|l|}{ No. per group } \\
\hline Baseline & 65 & 174 & \\
\hline 12-13.99 years & 43 & 163 & \\
\hline \multirow[t]{2}{*}{$14-15.99$ years } & 22 & 132 & \\
\hline & Mean $(95 \% \mathrm{Cl})$ & Mean $(95 \% \mathrm{Cl})$ & $P^{*}$ \\
\hline \multicolumn{4}{|l|}{ Age, years } \\
\hline Baseline & $7.3(7.0-7.5)$ & $7.8(7.6-8.0)$ & 0.001 \\
\hline $12-13.99$ years & $12.9(12.8-12.9)$ & $13.0(12.9-13.0)$ & 0.05 \\
\hline $14-15.99$ years & $14.9(14.7-15.0)$ & $14.9(14.9-15.0)$ & 0.38 \\
\hline \multicolumn{4}{|l|}{ Height, cm } \\
\hline Baseline & $125.6(124.2-127.0)$ & $127.2(126.3-128.1)$ & 0.06 \\
\hline $12-13.99$ years $^{\dagger}$ & $156.4(155.1-157.8)$ & $157.3(156.6-158.0)$ & 0.26 \\
\hline $14-15.99$ years $^{\dagger}$ & $168.5(166.5-170.4)$ & $171.2(170.4-172.0)$ & 0.01 \\
\hline \multicolumn{4}{|l|}{ Weight, kg } \\
\hline Baseline & $27.3(26.0-28.5)$ & $27.0(26.2-27.7)$ & 0.70 \\
\hline $12-13.99$ years $^{\dagger}$ & $47.6(45.3-49.8)$ & $51.3(50.2-52.5)$ & 0.003 \\
\hline $14-15.99$ years $^{\dagger}$ & $59.1(55.0-63.2)$ & $63.9(62.3-65.6)$ & 0.03 \\
\hline \multicolumn{4}{|l|}{$\mathrm{BMI}, \mathrm{kg} / \mathrm{m}^{2}$} \\
\hline Baseline & $17.0(16.5-17.6)$ & $16.5(16.2-16.9)$ & 0.14 \\
\hline $12-13.99$ years $^{\dagger}$ & $19.3(18.5-20.0)$ & $20.6(20.2-21.0)$ & 0.003 \\
\hline 14-15.99 years ${ }^{\dagger}$ & $20.5(19.2-21.8)$ & $21.7(21.2-22.2)$ & 0.09 \\
\hline \multicolumn{4}{|l|}{ Puberty, Tanner stage $e^{\ddagger}$} \\
\hline 12-13.99 years & $2.7(2.4-2.9)$ & $2.6(2.5-2.8)$ & 0.79 \\
\hline $14-15.99$ years & $3.6(3.2-3.9)$ & $4.0(3.8-4.1)$ & 0.03 \\
\hline \multicolumn{4}{|l|}{ Height velocity, $\mathrm{cm} /$ year $^{5}$} \\
\hline Baseline to 12-13.99 years & $5.8(5.5-6.1)$ & $5.7(5.6-5.8)$ & 0.68 \\
\hline Baseline to $14-15.99$ years & $5.8(5.5-6.1)$ & $6.1(6.0-6.2)$ & 0.06 \\
\hline \multicolumn{4}{|l|}{ Weight velocity, $\mathrm{kg} /$ year ${ }^{\varsigma}$} \\
\hline Baseline to 12-13.99 years & $4.1(3.6-4.6)$ & $4.6(4.3-4.8)$ & 0.14 \\
\hline Baseline to $14-15.99$ years & $4.4(3.7-5.1)$ & $5.1(4.9-5.4)$ & 0.04 \\
\hline
\end{tabular}

and recorded no refusals (estimated on the premise that children on longterm treatment are reviewed every 6 months). Ethics approval was granted by the Sydney West Area Health Service Human Research Ethics Committee. Parental informed consent was obtained, together with consent from all participating children.

Methylphenidate was the drug of first choice for boys with ADHD. Dexamphetamine was prescribed if the response to methylphenidate was suboptimal or as an alternative for boys with significant side effects. The dose of medication was titrated to the lowest dose giving a satisfactory therapeutic response.

Height and weight measurements were taken at study enrolment, and a review of medical records was undertaken to obtain each boy's baseline measurements (before starting treatment). Each boy was asked to identify his stage of puberty using Tanner staging with the help of pictures. ${ }^{6}$

For the controls, we used data from boys participating in the Nepean longitudinal study, an observational study originally designed to investigate the effects of birth size, body size and genes on blood pressure and bone mass. ${ }^{3-5}$ All were born at Nepean Hospital in western Sydney between 1989 and 1990 and had height and weight measurements available at 7-8 years of age and from one or both reviews at 13 and 15 years of age, when self-reported Tanner pubertal staging was also recorded. ${ }^{5}$

For subjects and controls, height was measured to the nearest $1 \mathrm{~mm}$ using a wall-mounted stadiometer, and weight was measured to the nearest $0.1 \mathrm{~kg}$ using electronic scales. All measurements were made without shoes and in light indoor clothing. Body mass index (BMI) was calculated as weight $/$ height ${ }^{2}\left(\mathrm{~kg} / \mathrm{m}^{2}\right)$. Measurements of height, weight and BMI were converted to age corrected $\mathrm{z}$ scores using the United States Centers for Disease Control and Prevention reference data using the LMS method. ${ }^{7,8}$

For analysis, the study cohort was stratified by age: 12.00-13.99 and 14.00-15.99 years. This was principally because the control data were clustered by age (Box 2). Growth parameters and pubertal stage of subjects and controls were compared using general linear modelling with age and baseline parameter $\mathrm{z}$ score (for growth data) as covariates.

Medication dose was expressed as methylphenidate equivalents, which were calculated by multiplying dexamphetamine dose by 2 . Medication dose effects on puberty and height velocity were assessed using Pearson's correlation analysis and general linear modelling, adjusting for potential confounders including duration of treatment, intermittent dosing, weight and age. All analyses were two-tailed and statistical significance was taken as $P<0.05$.

\section{Results}

Sixty-five boys were recruited (mean age, $13.53 \pm 1.09$ years; range, 12.02 15.88 years). The mean age of starting medication was $7.25 \pm 1.87$ years (range, 3.51-10.90 years) and mean treatment duration was $6.28 \pm 1.93$ years (range, 3.01-10.90 years).

At enrolment, 51 (78\%) were taking methylphenidate (mean dose, $46 \pm 17 \mathrm{mg} /$ day, $0.96 \pm 0.41 \mathrm{mg} / \mathrm{kg} /$ day; range, $18-90 \mathrm{mg} /$ day) and $14(22 \%)$ were on dexamphetamine (mean dose, $18.7 \pm 7.7 \mathrm{mg} /$ day, $0.34 \pm 0.15 \mathrm{mg} /$ $\mathrm{kg}$ /day; range, $7.5-30 \mathrm{mg} /$ day). Some children took medication intermittently, categorised as follows: $41 \%$ were taking the same dose of medication every day; $21 \%$ took medication on all schooldays and some medication on some non-school days; $34 \%$ took medication on school days only; and $3 \%$ took medication less frequently than every school day. Control growth data were available from 224 boys; 174 were included in this study 
because they had puberty data for at least one review.

When baseline measures were compared, the subjects before treatment were significantly younger than the controls aged 7-8 years (Box 2). There were no significant differences in height, weight or BMI after adjusting for age.

\section{Growth}

For the 43 subjects aged 12.00-13.99 years, the age-adjusted weights and BMIs were significantly lower than those of the controls $(P=0.003$ for both) (Box 2). There were no significant differences in age-adjusted height velocity or weight velocity.

For the 22 subjects aged 14.0015.99 years, after adjusting for age, the subjects were significantly shorter and lighter than the controls $(P=0.01$ and $P=0.03$, respectively) (Box 2 ). The mean age-adjusted weight velocity was significantly lower than the controls $(P=0.04)$ but there was no significant difference in age-adjusted height velocity.

\section{Puberty}

After adjusting for age, the 22 subjects aged 14.00-15.99 years reported significantly less advancement in their pubertal development $(P=0.03)$. There was no significant difference in stage of puberty at $12.00-13.99$ years of age.

\section{Effect of medication dose}

There was a significant inverse relationship between dose of medication (mg/day) and height velocity in the boys aged $14.00-15.99$ years $(r=0.47$; $P=0.03)$, which remained significant after adjusting for weight $(P=0.02)$ and age $(P=0.03)$ (Box 3$)$. There was no significant dose effect on height velocity in the boys aged 12.00-13.99 years. The stage of puberty showed a significant correlation with the height velocity in the boys aged 12.00-13.99 years and those aged 14.00-15.99 years $(\mathrm{r}=0.36 ; P=0.02$, and $r=0.45 ; P=0.03$, respectively), but there was no significant effect of the dose of medication on the stage of puberty in either age group. There was no independent effect of intermittent treatment or treatment duration on height velocity and no
3 Mean height velocity of 22 boys over an average of 7 years of treatment with stimulant medication to 14-15.99 years of age*

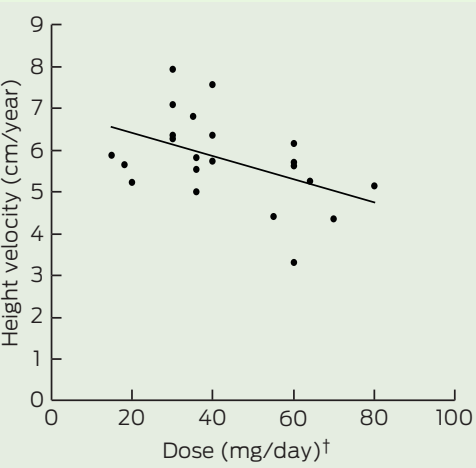

$* r^{2}=0.22 ; r=0.44 ; P=0.03 . \dagger$ Methylphenidate equivalent

significant difference between dexamphetamine and methylphenidate.

\section{Discussion}

We found that, in boys who had taken stimulant medication for ADHD for a minimum of 3 years until age 12.0013.99 years, there was lower weight but comparable growth in height and pubertal development compared with controls. Subjects aged 14.00-15.99 years were significantly behind the controls in their growth in height and pubertal development, with an inverse relationship between the dose of medication and height velocity. These findings suggest that stimulant medication delays the rate of maturation during puberty, including the timing of the peak height velocity, but not the onset of puberty.

The main strengths of this study were its longitudinal design, the relative consistency of the treatment history, and the duration of at least 3 years of treatment into adolescence. The high recruitment rate of eligible boys may be related to their positive therapeutic relationships with the study doctors (although eligibility was biased by the requirements for longterm treatment and baseline data, and our study may therefore not be representative of boys diagnosed with ADHD or who changed doctors). Another major strength was the quality and quantity of the control data drawn from a birth cohort from the same locality.

An important weakness of the study was its use of pubertal self- staging, which could be associated with lack of precision or observer bias. Imprecision would tend to predispose to a type II error, but we found a significant difference between groups despite the methodological limitations. Further, if there were observer bias, the same direction of bias would be anticipated in the subjects and controls and would be unlikely to result in spurious differences between groups. Although pubertal staging by the doctor might give more reliable data, it is probable that few adolescent boys would have agreed to this. A study comparing physician ratings with self-reported pubertal staging in boys has found $91 \%$ concordance. ${ }^{9}$

A further weakness was the lack of a control group of children with untreated ADHD. This meant that it was not possible to determine definitively whether differences in growth and physical development between the subjects and controls were due to ADHD or to treatment with stimulant medication. The dose relationship with the height velocity is highly suggestive of attenuation due to treatment. However, this finding could also be explained if children with more severe ADHD develop more slowly and if the dose of medication is only a marker of severity of ADHD.

We could find no longitudinal studies analysing the effect of stimulant medication on pubertal development. A cross-sectional study of 124 predominantly stimulant-treated boys with ADHD and 109 controls found no difference in the timing of the stages of puberty. ${ }^{10}$ Boys with ADHD in early puberty (Tanner stage 1-3) had shorter stature than boys without ADHD, but boys in late puberty (Tanner stage $4-5$ ) had normal heights, implying catch-up growth during puberty. Although there was no evidence of pubertal delay, there is the suggestion of compromised height during the earlier stages of puberty in the boys with ADHD. A comparable study of girls by the same research team showed no delay in growth or pubertal development. ${ }^{11}$ However, no information was given on the duration of treatment.

Our previous study of prepubertal children showed that during the first 2.5 years of treatment with stimulant medication, the height velocity was about $1 \mathrm{~cm} /$ year slower than 
expected. ${ }^{1}$ We therefore anticipated, but did not find, slower growth rates in the younger boys. This could be due to the onset of the adolescent growth spurt, which has a peak height velocity of around 7-9 cm/year and occurs between Tanner stages 3 and 4. ${ }^{12}$ The physiological increase of up to $4 \mathrm{~cm} /$ year that is found in normal boys during puberty - and which occurs with a variable age of onset, reaching higher levels in early developers - would rapidly obscure in our cohort of boys with ADHD any residual early effect of the stimulant on growth. By contrast, the boys aged 14.00-15.99 years with ADHD showed slower growth, as indicated by their shorter stature compared with the controls. We postulate that this was due to a delay in the adolescent growth spurt. This delay would become most apparent after the majority of the boys without ADHD reached their peak height velocity. The significant inverse correlation between height velocity and dose of medication identifies this as a medication effect. It is likely that the pubertal delay observed in the ADHD subjects aged 14.00-15.99 years is similarly attributable to treatment with stimulant medication, given the well recognised correlation between peak height velocity and stage of puberty. ${ }^{12}$ Delay in the peak height velocity could have adverse social implications, for example, in the context of sport, where size and strength may be important.

The lack of any significant effect of duration of treatment on growth or development was anticipated and is consistent with the notion that the negative impact of stimulant medication on growth has reached its maximum by 3 years. This could be reassuring for children who may have been on treatment for 7 years or more by the time they reach puberty. Similarly, intermittent treatment appeared to bestow no advantage for growth. This might be because the effect of eating more at the weekend, when medication is reduced, is balanced by increased appetite suppression during the early part of the week, when usual doses are restored.

The pattern of delay in the rate of physical maturation during puberty found in our study could have social implications for teenage boys with ADHD who may be less tall and less well developed than their peers. However, stimulant medication was also associated with a slower rate of weight gain.

To our knowledge, this is the first study to demonstrate delayed pubertal development in boys with ADHD compared with boys without ADHD, and to demonstrate a relationship between the dose of stimulant medication and the height velocity over an average of 7 years of treatment. This effect on growth appears to be distinct from the early attenuation observed in the first 3 years of treatment in other studies, because it was not observed in younger adolescents aged 12.0013.99 years. We postulate that stimulant treatment of 3 or more years is associated with a slower rate of physical development during puberty. In treating children with ADHD, it is important to use the lowest dose that is compatible with therapeutic efficacy, so that an adequate growth rate is maintained. Further study would be useful to determine the timing of peak height velocity and final adult stature in relation to the history of treatment with stimulant medication.

Acknowledgements: We thank the children and their families for agreeing to participate in this study. The stimulant growth study was supported by the Australian Women and Childrens Research Foundation. The Nepean longitudinal study was supported by National Health and Medical Research Council (NHMRC) grant 206501 and a Meat and Livestock Australia grant. Sarah Garnett was supported by an NHMRC Australian Clinical Research Fellowship from 2007 to 2010 (457225) and is currently supported by a Cancer Institute NSW Fellowship from 2011 to 2013 (10/ECF/2-11). The funding organisations had no role in the design and conduct of the study; collection, management, analysis and interpretation of the data; and preparation, review or approval of the manuscript.

Competing interests: No relevant disclosures.

Received 6 Jun 2012, accepted 5 Nov 2012.

1 Poulton A, Cowell CT. Slowing of growth in height and weight on stimulants: a characteristic pattern. J Paediatr Child Health 2003; 393 : 180-185.

2 Graber JA, Seeley JR, Brooks-Gunn J, Lewinsohn PM. Is pubertal timing associated with psychopathology in young adulthood? J Am Acad Child Adolesc Psychiatry 2004; 436: 718-726.

3 Garnett S, Cowell C, Bradford D, et al. Effects of gender, body composition and birth size on IGF-I in 7-and 8-year-old children. Horm Res 2000; 525: 221-229.

4 Garnett SP, Baur LA, Srinivasan S, et al. Body mass index and waist circumference in midchildhood and adverse cardiovascular disease risk clustering in adolescence. Am J Clin Nutr 2007; 863: 549-555.

5 Garnett SP, Cowell CT, Baur LA, et al. Increasing central adiposity: the Nepean longitudinal study of young people aged 7-8 to 12-13 y. Int J Obes 2005; 2911: 1353-1360.

6 Marshall WA, Tanner JM. Variations in the pattern of pubertal changes in boys. Arch Dis Child 1970; 45239: 13-23.

7 Cole TJ. The LMS method for constructing normalized growth standards. Eur J Clin Nutr 1990; 441: 45-60.

8 Kuczmarski RJ, Ogden CL, Guo SS, et al. 2000 CDC growth charts for the United States: methods and development. Vital Health Stat 11 2002; 246.

9 Duke PM, Litt IF, Gross RT. Adolescents' selfassessment of sexual maturation. Pediatrics 1980; 666: 918-920.

10 Spencer TJ, Biederman J, Harding M, et al. Growth deficits in ADHD children revisited: evidence for disorder-associated growth delays? J Am Acad Child Adolesc Psychiatry 1996; 3511: 1460-1469.

11 Biederman J, Faraone SV, Monuteaux MC, et al. Growth deficits and attention-deficit/ hyperactivity disorder revisited: impact of gender, development, and treatment. Pediatrics 2003; 1115 Pt 1: 1010-1016.

12 Ferrandez A, Carrascosa A, Audi L, et al. Longitudinal pubertal growth according to age at pubertal growth spurt onset: data from a Spanish study including 458 children ( 223 boys and 235 girls). J Pediatr Endocrinol Metab 2009; 228: $715-726$.

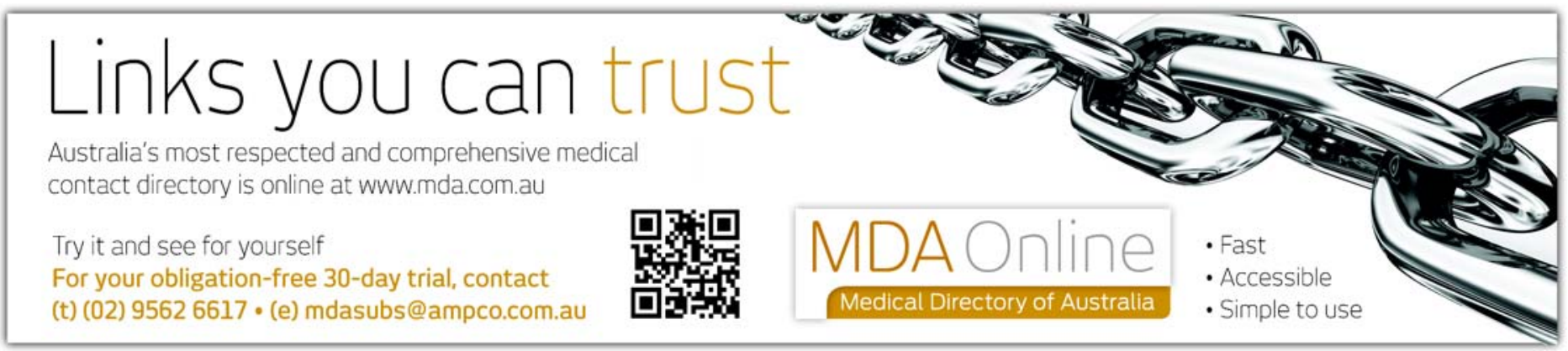

